\title{
Follow-up studies of children with birth defects in the Medical Birth Registry of Norway: A review
}

\author{
Rolv T. Lie and Rolv Skjærven \\ The Medical Birth Registry of Norway, Norwegian Institute of Public Health and \\ Section of Epidemiology and Medical Statistics, University of Bergen \\ Correspondence: Rolv T. Lie, Section for Epidemiology and Medical Statistics, Department of Public Health and Primary Health Care, \\ University of Bergen, Kalfarveien 31, N-5018 Bergen, Norway \\ E-mail: rolv.lie@smis.uib.no
}

\begin{abstract}
In two studies published from data in the Medical Birth Registry of Norway we described the survival, tendency to reproduce and subsequent recurrence of birth defects in offspring for children with a range of categories of birth defects. The studies were done separately for girls and boys. The current review summarizes data from these reports and makes some comparisons between boys and girls. More boys than girls are born with birth defects. The survival and tendency to reproduce showed great variation from milder to more serious types of defects, and this pattern was relatively consistent for boys and girls. The recurrence of birth defects in the offspring was almost entirely for a similar type of birth defect. For boys with birth defects, however, there was also a tendency to have offspring with other types of birth defects. A total of $0.5 \%$ of birth defects in the next generation was attributable to mothers who themselves had birth defects. For fathers with birth defects this number was higher (1.6\%) both because more boys were born with birth defects in the previous generation, but also because fathers pass on more birth defects to the next generation.
\end{abstract}

Few comprehensive population based follow-up studies of birth defects have been published. The Medical Birth Registry of Norway (MBRN) covers all births in Norway since 1967 . The registry records contain the unique person identification numbers for the child, the mother and the father of most births. This enables linkage within the registry of births of a child with data on survival and with records of that child's own offspring.

In two papers we utilized these unique opportunities to follow girls born with birth defects (Skjærven et al., 1999) and boys born with birth defects (Lie et al., 2001). Here we provide a brief review of these papers. Apparent differences between boys and girls are discussed.

\section{MATERIAL AND MethodS}

In the first paper (Skjærven et al., 1999) we followed a cohort of 459,433 live and stillborn female infants delivered in Norway between 1967 and 1982 in the MBRN. Altogether 8192 or $1.8 \%$ of the girls in the cohort had birth defects. In the second paper (Lie et al., 2001) we followed 486,207 live and stillborn male infants delivered in Norway between 1967 and 1982. The number of boys with birth defects was higher $(12,292$, or $2.5 \%)$ than in the cohort of girls. We linked these cohorts with data on survival up to 1992 . The proportion of babies with birth defects surviving within the follow-up was estimated and related to survival among non-affected babies (rate ratio presented as per cent in figures 1 and 2). Kaplan-Meier estimates of survival are presented in the original papers.
We identified all offspring of children in the cohorts through the MBRN. The proportion of individuals with birth defects who had reproduced was related to the proportion of non-affected individuals in the cohort who had reproduced (observed to expected ratio in figures 1 and 2). Further details on methods may be found in Skjærven et al., 1999 and Lie et al., 2001.

The first paper followed girls through 1997. Because boys tend to reproduce at a higher age than girls, the second cohort was followed though 1998 to provide a similar number of offspring. In the follow-up we probably also missed some offspring of boys, since information on father's identity was missing in $5.6 \%$ of birth records in more recent years.

Registration of birth defects in MBRN is not complete and varies across birth defects categories (Lie et al., 1994). We classified birth defects into 24 major categories, basically corresponding to ICD-8 subsections (details are given in Lie et al., 1994).

\section{RESUlts}

Girls with birth defects had a lower survival than girls without birth defects $(80 \%$ vs. 98\%). A similar but smaller difference was seen for boys ( $84 \%$ vs. 97\%). The higher survival of boys with birth defects may indicate that boys are affected by more minor defects.

Figure 1 shows the survival of girls with different categories of birth defects relative to girls without birth defects. As expected, serious defects like neural tube defects and defects of vital organs had reduced survival. This pattern of reduced survival was similar to that 
of boys with birth defects (Figure 2).

Within the cohort of girls we identified that $27 \%$ had reproduced by 1997 . For the cohort of boys the corresponding proportion was $17 \%$ by 1998 .

Overall the tendency to survive correspond with the tendency to have offspring across the different categories of defects both for girls and boys. There were some exceptions, though. Children with clubfoot had almost no reduced survival in our data, but appeared to have a somewhat reduced tendency to have offspring (at least within our limited follow-up). This tendency was similar for boys and girls. Cleft lip also had almost normal survival, but appeared to have reduced tendency to reproduce only among boys.

The proportion of children with birth defects was $3.8 \%$ for mothers who had birth defects compared with $2.4 \%$ for children of mothers without birth defects, for a relative risk of $1.6(95 \% \mathrm{CI}: 1.2-2.0)$. For males with birth defects the proportion of offspring with defects was $5.1 \%$ and for males without birth defects the proportion in offspring was $2.1 \%$. The lower overall proportion was probably due to missing father's information on some still births. Thus, relative risk of recurrence was 2.4 (95\% CI: 1.7-2.8) for fathers. This overall recurrence from father to offspring was significantly higher than from mother to offspring $(p=0.03)$ when adjustment is made for a slightly different composition of defects for offspring of fathers and mothers with birth defects.
Only $0.5 \%$ of birth defects in the next generation was attributable to mothers who themselves had birth defects. For fathers with birth defects the attributable risk was higher $(1.6 \%)$ both because more boys were born with birth defects in the previous generation, but also because fathers tend to pass on more birth defects to the next generation.

Tables 1 and 2 show recurrence of a similar type of defects for mothers and fathers with cleft lip, clubfoot or limb defects and as a weighted average across all 24 categories of defects. The tendency of specific defects to recur did not appear to be very different for mother and father. In tables 3 and 4, the tendency of "recurrence" of a different type of defect is shown. Motheroffspring recurrence appeared to be almost entirely of the same type of defects, while recurrence from father to offspring was also increased for other types of defects than the type recorded on the father. This more unspecific recurrence from father to offspring may explain the overall higher recurrence from father to offspring than from mother to offspring.

\section{Discussion}

The potential for constructing population based follow-up studies within the MBRN is great, and is still increasing. Since the registry covers all births in the Norwegian population since 1967 , the registry now covers almost the complete reproductive history of

Figure 1. Survival and childbearing of females with various categories of birth defects relative to females without birth defects. Survival of females without birth defects is set to $100 \%$ and their childbearing proportion is set to 1.0.

Females: Survival and Childbearing by Category of Birth Defect

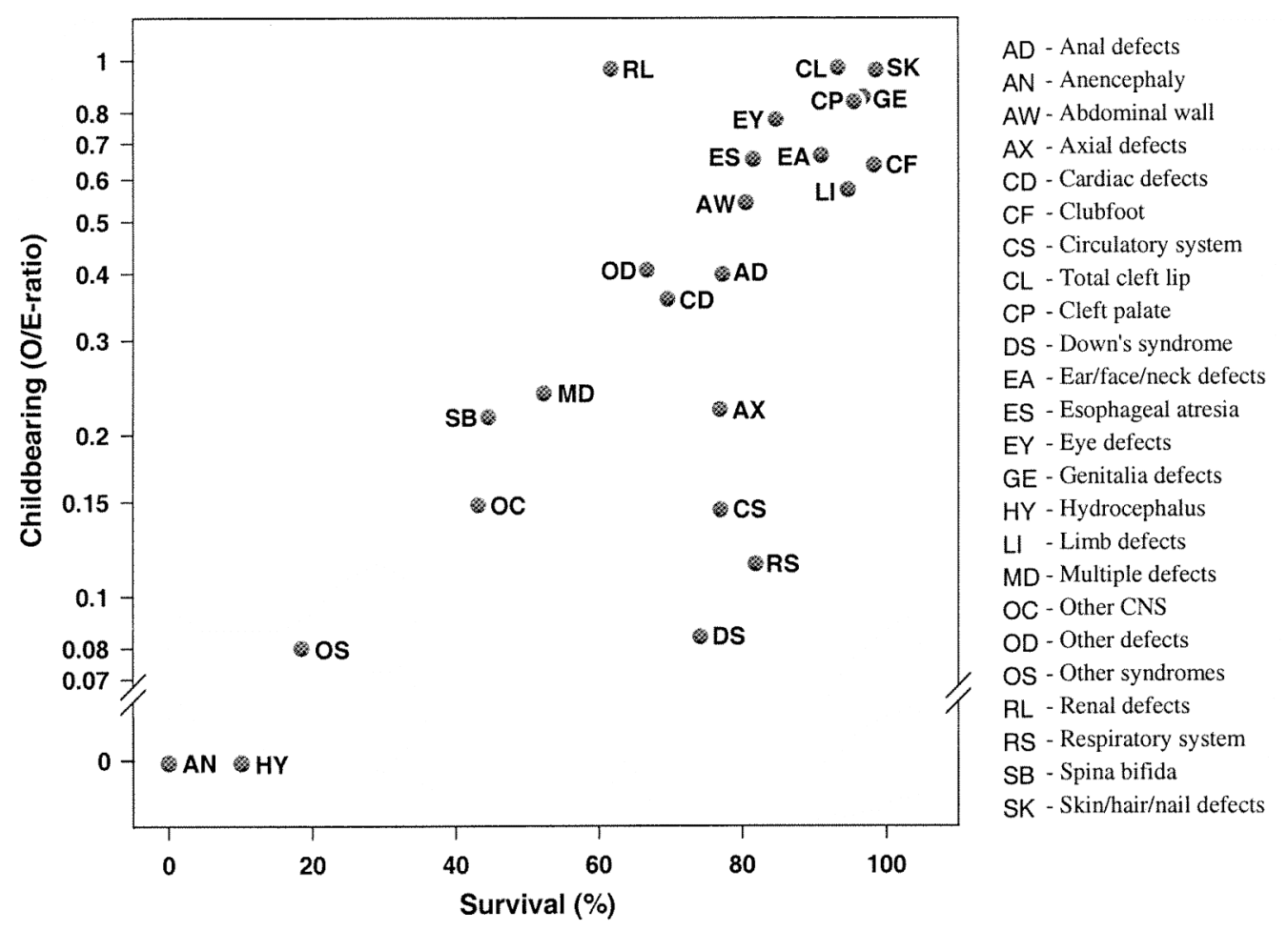


children born in the first years of the registry forty years ago.

One main limitation of the two studies reviewed here is the incomplete follow-up, since they only cover the first years of the reproductive career of the cohorts. This limitation will be less important in future studies. The number of observed recurrences is also relatively small for several categories of defects. Another limitation is the incomplete identification of fathers in the follow-up. It is important that the registry cover as many fathers as possible and that selective loss of information for births affected by defects or other complications is avoided.

The impact of these problems on our major findings may be small. Future studies will be able to give an even better description of the cohorts and clarify whether the differences we see between boys and girls with defects are real.

The higher recurrence of defects from father to offspring than from mother to offspring is particularly intriguing. It will be valuable to see if this tendency is confined to particular categories of defects. To do this, we need larger cohorts and longer follow-up. In general, a higher impact of paternal inheritance may be explained by so-called genomic imprinting. Evidence of genomic imprinting may have great implications for search for genetic causes of birth defects.

Reduced tendency to reproduce for males and females with birth defects may be caused by both biolo- gical and social factors. Still, problems with reproduction may be more pronounced for cases that are more severely affected within each category of defects. The strong selection across generations for some serious categories of defects should therefore be considered when recurrence across generations is compared with recurrence between siblings (Lie et al., 1994). Sibrecurrence is not conditional on survival and reproduction of the index case. Analyses that pool those recurrences between these so called first-degree relatives (that share $50 \%$ of their alleles) may have problems if parent-offspring recurrence is strongly affected by selection.

Another interesting finding in these studies was the low number of birth defects in the next generation that could be attributed to the parents having had birth defects. This proportion was as low as around $2 \%$. Males and females with birth defects had overall only a moderately reduced tendency to have children compared to other males and females. Still, their contribution of defects to the next generation appears almost negligible.

\section{ACKNOWLEDGEMENT}

Thanks to our friend and colleague Dr. Allen Wilcox for great and continuing collaboration through many years, collaboration that among other things resulted in the papers reviewed here.

Figure 2. Survival and childbearing of males with various categories of birth defects relative to males without birth defects. Survival of males without birth defects is set to $100 \%$ and their childbearing proportion is set to 1.0.

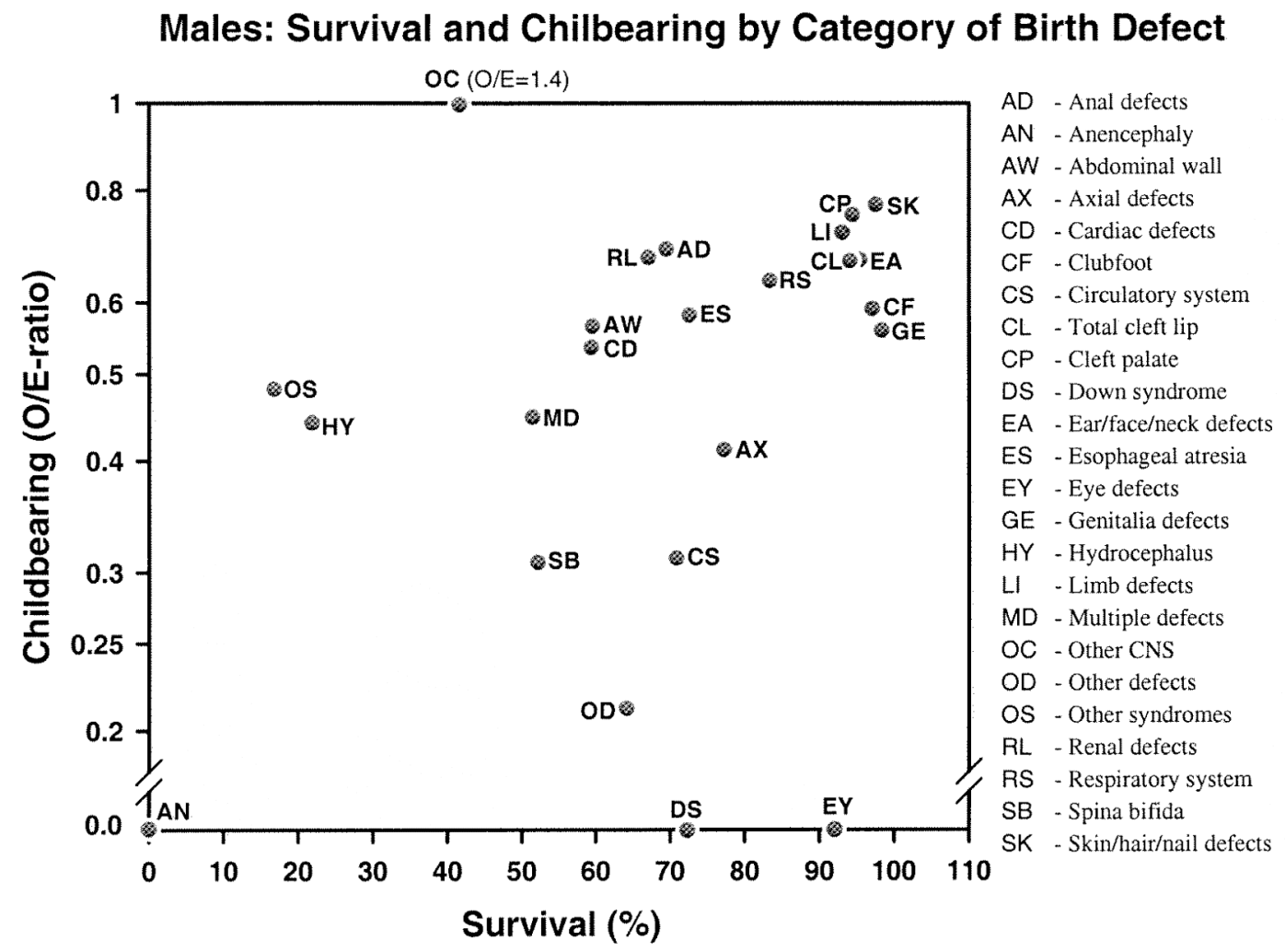


Table 1. Recurrence of a similar birth defect from mother to child for selected categories of defects.

\begin{tabular}{lccc}
\hline Defect in mother & Observed (O) & Expected (E*) & O/E Ratio (95\% CI) \\
\hline Cleft Lip & 7 & 0.20 & $38(16-77)$ \\
Clubfoot & 15 & 2.8 & $5.5(3.2-9.1)$ \\
Limb defects & 2 & 0.37 & $5.6(0.9-18.7)$ \\
\hline Total $^{\dagger}$ & 26 & 3.9 & $6.8(4.5-10.0)$ \\
\hline
\end{tabular}

* The expected number was calculated from offspring of fathers without birth defects

$\dagger$ The total include all 24 categories of defects

Table 2. Recurrence of a similar birth defect from father to child for selected categories of defects.

\begin{tabular}{lccc}
\hline Defect in father & Observed (O) & Expected (E*) & O/E Ratio (95\% CI) \\
\hline Cleft Lip & 6 & 0.16 & $38(14-93)$ \\
Clubfoot & 4 & 1.17 & $3.4(0.9-9.0)$ \\
Limb defects & 3 & 0.25 & $12(2.5-37)$ \\
\hline Total $^{\dagger}$ & 21 & 3.22 & $6.5(4.0-10.4)$ \\
\hline
\end{tabular}

* The expected number was calculated from offspring of fathers without birth defects

$\dagger$ The total include all 24 categories of defects

Table 3. Recurrence of dissimilar birth defects from mother to child for selected categories of defects.

\begin{tabular}{lccc}
\hline Defect in mother & Observed (O) & Expected (E*) & O/E Ratio (95\% CI) \\
\hline Cleft Lip & 4 & 3.4 & $1.2(0.4-2.9)$ \\
Clubfoot & 17 & 13 & $1.3(0.8-2.1)$ \\
Limb defects & 5 & 5.3 & $0.9(0.3-2.2)$ \\
\hline Total $^{\dagger}$ & 36 & 34.6 & $1.0(0.7-1.4)$ \\
\hline
\end{tabular}

* The expected number was calculated from offspring of fathers without birth defects

$\dagger$ The total include all 24 categories of defects

Table 4. Recurrence of dissimilar birth defects from father to child for selected categories of defects.

\begin{tabular}{lccc}
\hline Defect in father & Observed (O) & Expected (E*) & O/E Ratio (95\% CI) \\
\hline Cleft Lip & 6 & 2.29 & $2.6(1.0-6.1)$ \\
Clubfoot & 6 & 5.57 & $1.1(0.4-2.4)$ \\
Limb defects & 6 & 3.67 & $1.6(0.6-3.7)$ \\
\hline Total $^{\dagger}$ & 43 & 23.7 & $1.8(1.3-2.5)$ \\
\hline
\end{tabular}

* The expected number was calculated from offspring of fathers without birth defects

$\dagger$ The total include all 24 categories of defects

\section{REFERENCES}

Lie RT, Wilcox A, Skjærven R. A population-based study of recurrence risks of birth defects. N Engl J Med 1994; 331: $1-4$.

Lie RT, Heuch I, Irgens LM. Maximum likelihood estimation of the proportion of congenital malformations using double registration systems. Biometrics 1994; 50: 433-44.

Lie RT, Wilcox AJ, Skjærven R. Survival and reproduction among males with birth defects and the risk of recurrence in their children. JAMA 2001; 285: 755-60.

Skjærven R, Wilcox AJ, Lie RT. A population-based study of survival and childbearing among female subjects with birth defects and the risk of recurrence in their children. N Engl J Med 1999; 340: 1057-62. 